\title{
Microwatts continuous-wave pumped second harmonic generation in few- and mono-layer GaSe
}

\author{
Xue-Tao Gan ${ }^{1}$, Chen-Yang Zhao ${ }^{1}$, Si-Qi Hu${ }^{1}$, Tao Wang ${ }^{2}$, Yu Song ${ }^{1}$, Jie Li ${ }^{2}$, Qing-Hua Zhao' Wan-Qi Jie ${ }^{2}$ \\ and Jian-Lin Zhao ${ }^{1}$
}

We demonstrate the first achievement of continuous-wave (CW) pumped second harmonic generation (SHG) in few- and monolayer gallium selenide (GaSe) flakes, which are coated on silicon photonic crystal (PC) cavities. Because of ultrahigh second order nonlinearity of the two-dimensional (2D) GaSe and localized resonant mode in the PC cavity, SHG's pump power is greatly reduced to microwatts. In a nine-layer GaSe coated PC cavity, while the optical power inside the GaSe flake is only $1.5 \%$ of that in the silicon PC slab, the SHG in GaSe is more than 650 times stronger than the third harmonic generation in silicon slab, indicating 2D GaSe's great potentials to strengthen nonlinear processes in silicon photonics. Our study opens up a new view to expand 2D materials' optoelectronic applications in nonlinear regime and chip-integrated active devices.

Light: Science \& Applications (2018) 7, 17126; doi:10.1038/Isa.2017.126; published online 12 January 2018

Keywords: gallium selenide; photonic crystal cavity; second harmonic generation; two-dimensional materials

\section{INTRODUCTION}

Two-dimensional (2D) materials with layered structure, such as graphene, black phosphorus, transition metal dichalcogenides and gallium monochalcogenides, have attracted much recent attentions to complement silicon electronics and optoelectronics potentially ${ }^{1-3}$. Second harmonic generation (SHG), normally achieved in noncentrosymmetric materials, as one of the strongest nonlinear processes has been widely employed in signal and image processing, laser industries and optical spectroscopies ${ }^{4,5}$. In virtue of shapeable electronic structures in the atomic layered crystals, SHGs in 2D materials occupy a variety of distinct characteristics. For instance, while the bulk $\mathrm{MoS}_{2}$ and $h$-BN have centrosymmetry, their finite slices with odd-layer thicknesses could yield moderately strong SHG, especially for the mono-layers with pure noncentrosymmetric $\mathrm{D}_{3 h}$ space group ${ }^{6-8}$. In contrast, SHGs were observed in even-layered $1 \mathrm{~T}^{\prime} \mathrm{MoTe}_{2}$ determined by the broken inversion symmetry9. SHGs in $2 \mathrm{D}$ materials also indicate unusually large second order nonlinear susceptibilities, which are nearly three orders of magnitude higher than other common nonlinear crystals ${ }^{10,11}$. At 2D crystals' boundaries, variations of electronic structure result in even stronger SHG with onedimensional edge state, facilitating direct optical imaging of crystal orientations and straightforward reveals of kinetic nature of grain boundary formation in as-grown mono-layers ${ }^{12,13}$. In addition, the strong exciton charging effect in mono-layer semiconductor fieldeffect transistor allows for exceptional control over the oscillator strengths between the exciton and trion resonances, enabling the electrically controlled nonlinear susceptibilities by an order of magnitude and counter-circular resolved SHG spectrum ${ }^{14}$.

During the past few years, 2D materials have been widely reported for promising optoelectronic devices including photodetectors, modulators, and light emission devices ${ }^{15-21}$. If $2 \mathrm{D}$ materials' extraordinary SHGs were exploited further, their optoelectronic applications might be greatly extended into nonlinear regimes for coherent light source generations, image processing, ultrafast laser engineering and so on. However, to the best of our knowledge, the previously demonstrated SHGs in 2D materials all required pulsed laser sources with high peak power (larger than $100 \mathrm{~mW}$ ). For practical applications, continuouswave (CW) pumped SHG by means of simple, low-power and lowcost light sources, such as semiconductor laser diodes, would be highly desirable.

In this article, we report it is possible to achieve strong SHG in atomically layered GaSe using a low-power CW laser. GaSe is a wellknown nonlinear crystal in the spectral range from visible to terahert $\mathrm{z}^{22}$. It has three most important classifications according to the layer stacking order, that is, $\beta$-GaSe, $\gamma$-GaSe and $\varepsilon$-GaSe. Here, we employ $\varepsilon$-GaSe, whose stacking sequence leads to the absence of inversion center for arbitrary layer thickness ${ }^{23}$. Hence, the achievement of SHG in GaSe has no layer dependence. In addition, GaSe has pronounced second order nonlinearity in its primitive layer consisting of two atomic layers of Ga sandwiched between two atomic layers of Se, which is 1-2 orders of magnitude larger than that of mono-layer $\mathrm{MoS}_{2}{ }^{11}$. By integrating few- and mono-layer GaSe flakes with silicon photonic crystal (PC) cavities to enable an effective interaction

${ }^{1}$ MOE Key Laboratory of Space Applied Physics and Chemistry and Shaanxi Key Laboratory of Optical Information Technology, School of Science, Northwestern Polytechnical University, Xi'an 710072, China and ${ }^{2}$ State Key Laboratory of Solidification Processing, Northwestern Polytechnical University, Xi'an 710072, China Correspondence: XT Gan, Email: xuetaogan@nwpu.edu.cn; JL Zhao, Email: jlzhao@nwpu.edu.cn

Received 24 March 2017; revised 23 August 2017; accepted 25 August 2017; accepted article preview online 28 August 2017

The accepted article preview was available with the details: Light: Science \& Applications (2018) 7, e17126; doi: 10.1038/lsa.2017.126 
between GaSe and cavity's evanescent field, a telecom-band CW laser is strong enough to pump SHG in the $2 \mathrm{D}$ GaSe, and the required power could be reduced down to only few microwatts. In addition, we observe the cavity-enhanced SHG in a nine-layer GaSe is more than two orders of magnitude stronger than the cavity-enhanced third harmonic generation (THG) in a thick silicon slab. It indicates GaSe's high second order nonlinearity could enable significant chipintegrated nonlinear effects. Therefore, integration of 2D GaSe, with high nonlinearity and negligible linear and nonlinear losses in telecom-band, onto nanophotonic structures may pave a new way towards developing high-performance chip-integrated nonlinear devices based on second order nonlinearity, such as on-chip frequency-conversion laser sources ${ }^{24}$, optical autocorrelator ${ }^{25}$, entanglement photon-pair generations ${ }^{26}$.

\section{MATERIALS AND METHODS}

We fabricated PC cavities on a silicon-on-insulator wafer with a 220-nm-thick top silicon layer (Supplementary Information), whose fabrication process is Complementary Metal Oxide Semiconductor (CMOS)-compatible and the integrated devices have potentials for future on-chip optical interconnects. Also, silicon has no second order nonlinearity, ensuring the measured second harmonic signal is purely from the GaSe layer without a background from the bulk silicon substrate. The exfoliated GaSe flake is integrated onto the PC cavity using a dry transfer method (Supplementary Information) ${ }^{27}$. Figure 1a displays an optical microscope image of the fabricated device with a large few-layer GaSe flake covering the cavity. The uniformity and thickness of the GaSe flake are examined using an atomic force microscopy (AFM), as shown in Figure 1b. The profile heights over the PC air-holes indicate conformal contact between the few-layer flake and the PC cavity with the dry transfer technique, which promises the effective interaction between GaSe and cavity evanescent field. The thickness of the transferred GaSe is examined as $7.8 \mathrm{~nm}$, corresponding to a layer number of nine by assuming the mono-layer thickness of $0.85 \mathrm{~nm}^{28}$. The photoluminescence (PL) of the transferred nine-layer GaSe is evaluated with a pump of 532-nm CW laser, as shown in the inset of Figure 1a. Because of the direct-to-indirect and broadening bandgap transition in the few-layer GaSe, the PL peak locates at $587 \mathrm{~nm}$ with a greatly reduced quantum yield. To achieve small mode volume and high-coupling efficiency of the PC cavity, which is crucial for cavity-enhanced $\mathrm{SHG}^{25,29-32}$, we design the cavity defect into a point-shifted type with shrunk air-holes ${ }^{33}$, as shown in the scanning electron microscope (SEM) image of Figure 1c. Pored holes in the GaSe layer over several PC air-holes are induced by the accelerated electron beam, which is witnessed during the SEM measurements.

The experimental measurements over the GaSe-PC cavity are implemented using a vertically coupled cross-polarization microscope (Supplementary Information) $^{34}$. The orthogonal polarizations of the excitation laser and the collection signal are achieved using a polarized beam splitter (PBS). A half wave plate (HWP) is inserted between the PBS and the objective lens to control the laser polarization with respect to the axis of the $\mathrm{PC}$ cavity. To evaluate resonant modes of the PC cavity, we illuminate the cavity with a CW tunable narrowband laser and monitor its reflection using a telecom-band photodiode. Cavity reflection spectra could be obtained by scanning the laser wavelength with a step of $0.005 \mathrm{~nm}$.

\section{RESULTS AND DISCUSSION}

The red and green dotted lines in Figure 1d display the same resonant mode of the PC cavity measured before and after the integration of GaSe, and the black dashed lines are Lorentzian fittings. After the
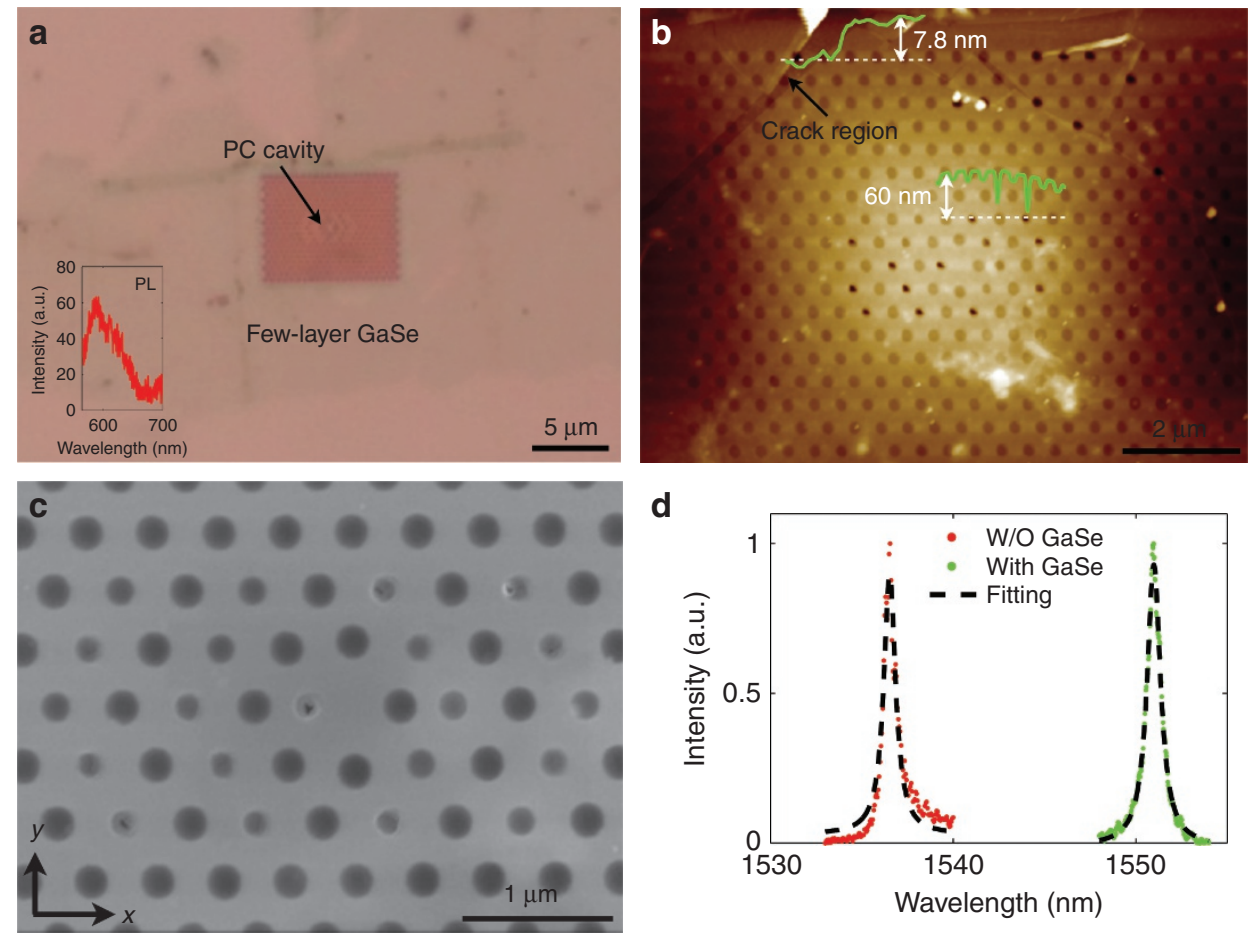

Figure 1 (a) Optical microscope image of the GaSe-PC cavity with the GaSe layer shown as light green flakes. Inset displays the PL spectrum of the ninelayer GaSe flake. (b) AFM image of the GaSe-PC cavity, where the thickness of the GaSe layer is indicated at the location of a crack region with a value of $7.8 \mathrm{~nm}$ and the dashed lines indicate the locations where the height distributions are measured. (c) SEM image of the GaSe-PC cavity. (d) Cavity's reflection spectra of the resonant mode before and after the integration of GaSe. 
integration, the GaSe layer works as a positive perturbation of the dielectric function around the resonant mode, which shifts the resonant wavelength from 1536.5 to $1551.0 \mathrm{~nm}$. It also weakens the confinement of the resonant mode, lowering the $Q$ factor from $\sim 2000$ to $\sim 1750$. These variations of the resonant mode are confirmed using three-dimensional finite element simulations (COMSOL Multiphysics) with the refractive index and thickness of the GaSe layer chosen as 2.8 and $7.8 \mathrm{~nm}^{35}$.

To implement the cavity-enhanced SHG measurement, we tune the laser wavelength as $1551 \mathrm{~nm}$ to excite the cavity's resonant mode, and the reflected resonant signal is filtered out using a short pass dichroic mirror (Supplementary Information). The frequency conversion signal scattered from the cavity is monitored using a $0.5-\mathrm{m}$ spectrometer mounted with a cooled silicon camera. Figure $2 \mathrm{a}$ displays an obtained spectrum, when the pump power is $0.5 \mathrm{~mW}$ measured after the objective lens. At the wavelength of $775.5 \mathrm{~nm}$, a strong peak is observed, corresponding to the second harmonic signal of the pump laser. Another weak peak arises as well at $517 \mathrm{~nm}$, equaling to the THG wavelength of the pump laser.

Before we transfer the GaSe layer, we examine the harmonic generations from the bare silicon PC cavity as well, which presents the similar THG peak intensity but no SHG peak. As reported in Ref. 31 , resonantly enhanced SHG in a bare silicon PC cavity is also possible due to the surface second order nonlinear process. However, this SHG signal is about two orders of magnitude weaker than the cavity-enhanced $\mathrm{THG}^{31}$, which is a possible reason for the failed observation of SHG in our bare PC cavity. Hence, the obtained strong SHG from the GaSe-PC cavity is due to the top GaSe layer. Also, the THGs measured before and after integration of GaSe have no noticeable power variation, indicating the THG in Figure 2a mainly results from the third order nonlinear process in the bulk silicon slab. Comparing SHG and THG of the GaSe-PC cavity, the SHG peak is more than 650 times stronger than the THG peak. From the resonant mode simulation, we calculate the optical power distributions in the GaSe-PC cavity, indicating the power inside the nine-layer GaSe flake is only $1.5 \%$ of that in the bulk silicon slab. The remarkably strong SHG signal is in consistent with the ultrahigh second order nonlinear susceptibility of $\mathrm{GaSe}^{11,22,28}$.

We also evaluate the pump power dependence of the SHG by varying the laser power, as shown in the log-log plot of Figure $2 \mathrm{~b}$. The dashed line indicates a fitting with a slope of 2.01, verifying the quadratic power dependence of the cavity-enhanced SHG. By measuring the cavity reflection of the on-resonance laser, and combining the far-field collection efficiency of the resonant mode $(\sim 40 \%)^{33}$, the coupling-in efficiency of the pump laser is estimated as $6 \%$. Therefore, even for a $0.1 \mathrm{~mW}$ pump illuminating on the PC cavity, the power coupled into the cavity is $<10 \mu \mathrm{W}$, which can still generate a detectable SHG.

For the previously reported SHG in mono-layer or few-layer 2D materials, pulsed lasers are employed to achieve high peak pump power and increase the SHG output signal. In our GaSe-PC cavity, the successful observation of SHG with a CW pump could be attributed to the strong enhancement of the pump power coupled into the cavity. To verify this, we acquire the SHG powers as we tune the pump laser wavelength cross the cavity resonance, as plotted in Figure $2 \mathrm{c}$. As the laser wavelength is away from the resonance, the SHG signal is nearly not detectable due to the weak light-matter interaction. For the onresonance pump, the laser power coupled into the cavity is enhanced by a factor proportional to $Q$, and the enhancement over the second order nonlinear process has a factor proportional to $Q^{2}(\operatorname{Refs} 25,29$,
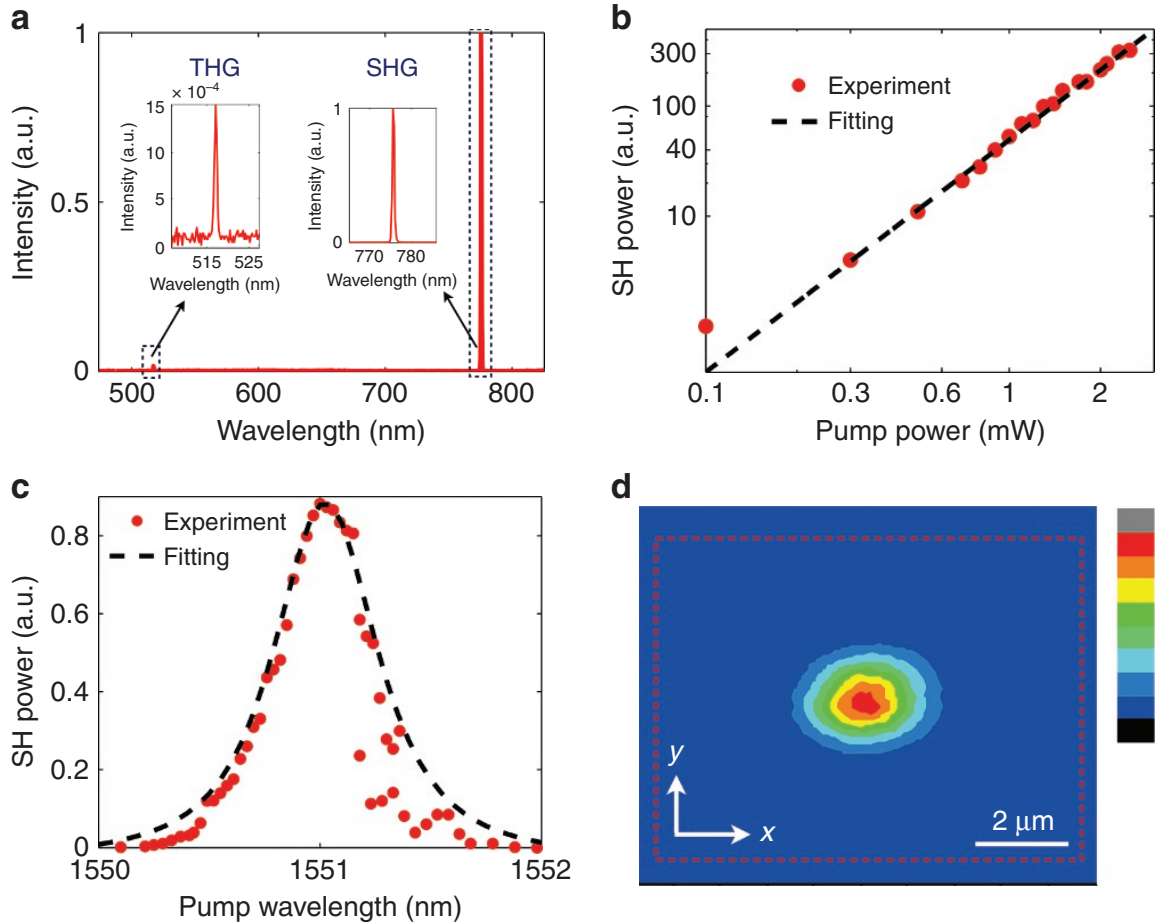

d

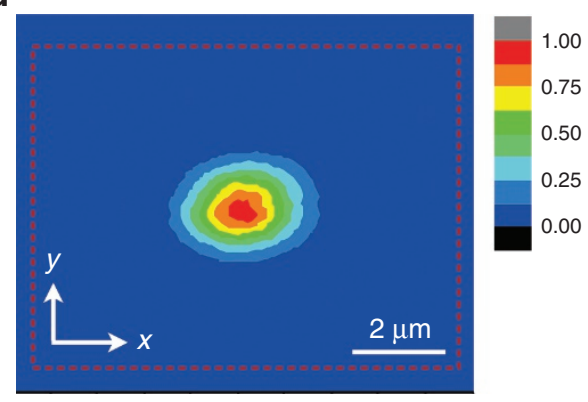

Figure 2 (a) Spectrum of the cavity's scattering signal with a $1551 \mathrm{~nm}$ pump laser, which has two signals at 775.5 and $517 \mathrm{~nm}$ corresponding to SHG and THG, respectively. (b) Pump power-dependence of the cavity-enhanced SHG with a fitting slope of 2.01, where the pump power is measured after the objective lens. (c) Cavity-enhanced SHG spectrum when the pump wavelength is tuned cross the resonance, where the dashed line is a squared Lorentzian fitting curve of the cavity resonant mode. (d) Spatial mapping of the cavity-enhanced SHG, where the PC boundary is indicated by the dashed line. 
31). Hence, the SHG spectrum could be very well described by the squared Lorentzian lineshape, which is used to fit the fundamental resonance shown in Figure 1d.

The cavity-enhanced SHG is verified as well using a spatial mapping of the SHG with an on-resonance pump, as shown in Figure 2d. The device is mounted on a $2 \mathrm{D}$ piezo-actuated stage with a moving step of $100 \mathrm{~nm}$, and the generated second harmonic signal is measured using a photomultiplier tube (PMT). As the cavity-enhanced SHG is pumped by the evanescent field of the cavity resonant mode, efficient SHG can only be observed when the pump laser couples into the cavity. Therefore, the spatial position of the detected SHG is determined by cavity's coupling-in region. For the modified pointshifted cavity, light could vertically couple into the cavity effectively around the modification region ${ }^{33}$, which has a dimension about $2.5 \times 2 \mu \mathrm{m}^{2}$. In the SHG mapping, a similar area with strong signal is observed. Outside the cavity mode-coupling region, the GaSe layer is only pumped by the vertically illuminated laser, which is too weak to yield observed SHG for a CW pump.

Because of the optical losses from various optical components in the microscope setup, as well as the absorption by the silicon substrate, it is difficult to evaluate the absolute SHG power enhanced by the cavity. To evaluate the cavity-enhancement factor, we switch the CW laser into a pulsed laser to pump the GaSe-PC cavity, with pumping wavelength (at $1560 \mathrm{~nm}$ ) being off-resonance from the cavity mode. In this regime, both the on-resonance CW pump and the off-resonance pulsed pump share the same excitation and collection optical paths, as well as the same location of GaSe layer to maintain the dielectric environment of the SHG emission. For a $2.8 \mathrm{nW}$ SHG measured by the PMT, the required powers for the $\mathrm{CW}$ laser and pulsed laser are measured as 2.5 and $1.56 \mathrm{~mW}$ (averaged power), respectively. For both the CW and pulsed laser excitations, the SHG process strongly depends on the strength of electric field. With parameters of the pulsed laser, we can calculate the effective electrical field used to generate SHG in GaSe. Combining with the experimental results and the calculations, we can estimate the enhancement factor as 612 (Supplementary Information).

In Figure 3a, we display the simulated electric fields of the resonant mode located at the GaSe layer, decomposed into the two orthogonal components $E_{x}$ and $E_{y}$. Because the odd-symmetry of $E_{y}$ would generate a far-field pattern splitting into a large oblique angle, the
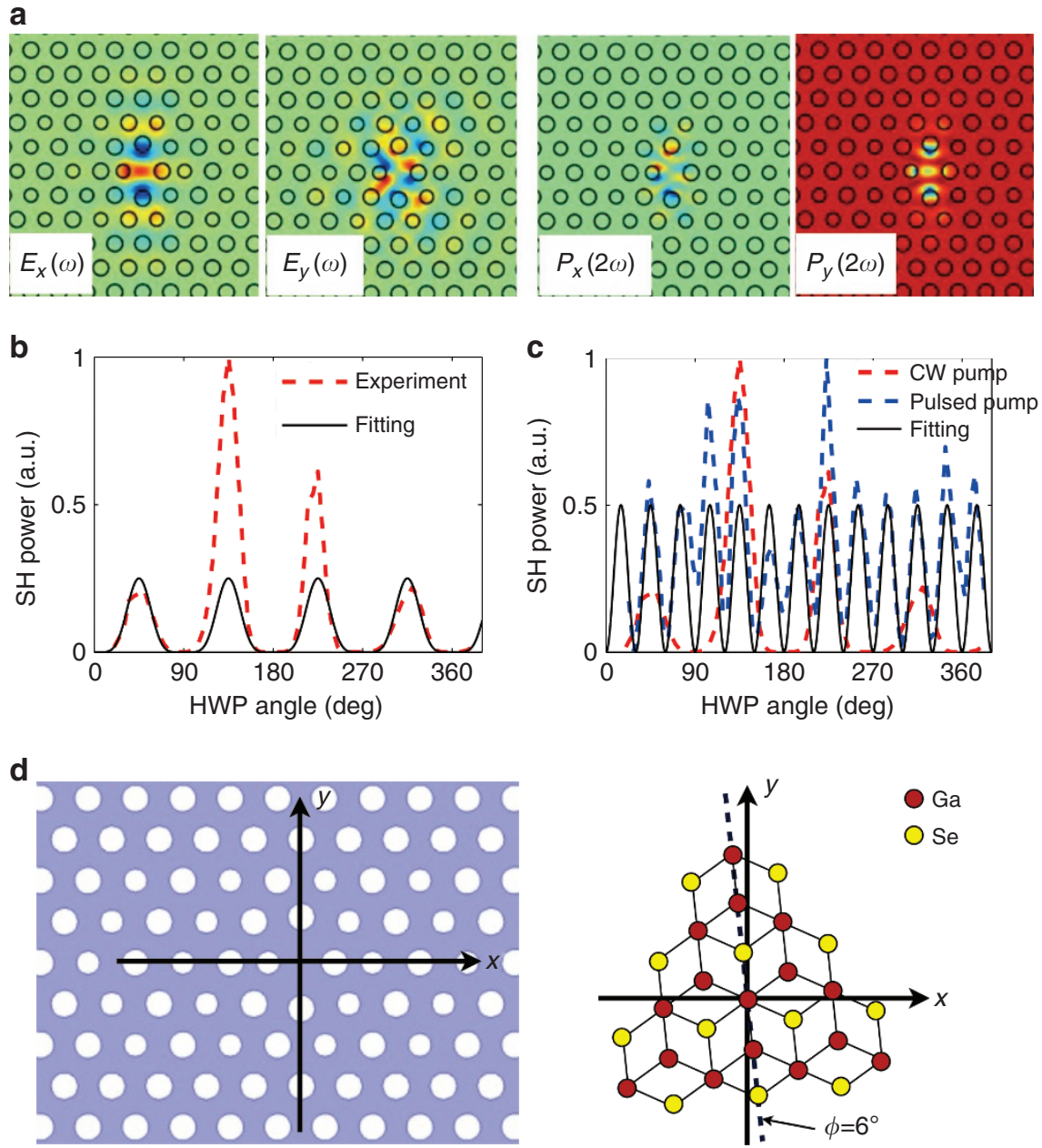

Figure 3 (a) Simulated electric fields $\left(E_{x}\right.$ and $\left.E_{y}\right)$ of the resonant mode and the corresponding nonlinear polarizations $\left(P_{x}\right.$ and $\left.P_{y}\right)$ calculated from the nonlinear susceptibility matrix and crystal orientation of GaSe. (b) Polarization-dependence of the cavity-enhanced SHG. The black solid line is a fitting of $\sin ^{6}(2 \theta)$, where $\theta$ is the angle between the HWP's fast axis and $y$-axis of the PC cavity. (c) Polarization-dependences of the SHG pumped by the on-resonance CW laser and off-resonance pulsed lasers. The SHG pumped by the off-resonance pulsed laser is fitted by a function of $\sin ^{2} 6\left(\theta+3^{\circ}\right)$, indicated by the black solid line. (d) Alignment of the GaSe crystal orientation with respect to the axes of the PC cavity. 
coupling between the cavity mode and a $y$-polarized far-field is very small. For a light with $x$-polarization, its far-field coupling with the resonant mode is high due to the vertically directed far-field pattern of $E_{x}$. Therefore, when an on-resonance laser focuses on the cavity, only the $x$-polarized component can couple into the cavity effectively. If the laser polarization is changed by rotating the HWP, the power coupled into the cavity is proportional to $\sin ^{2}(2 \theta)$, where $\theta$ is the angle between the HWP's fast-axis and the $y$-axis of the PC cavity. And the cavityenhanced SHG, which is proportional to the square of the coupled power, should follow a function of $\sin ^{4}(2 \theta)$. Considering the crosspolarization of the experimental setup, the vertically scattered SHG after the HWP is then projected onto the output polarization direction, and the finally collected SHG has a function of $\sin ^{6}(2 \theta)$, as plotted in Figure 3b. Here, the four peaks have different maximum values. We attribute it to the imperfection of the HWP at the SHG wavelength (the achromatic wavelength range of the HWP is 1200-1600 nm).

By switching the on-resonance CW laser into the off-resonance pulsed laser (at $1560 \mathrm{~nm}$ ), we observe another pump polarizationdependent SHG from the GaSe-PC cavity by rotating the HWP, as shown in Figure 3c. A fitting with a function of $\sin ^{2} 6\left(\theta+3^{\circ}\right)$ is obtained, which is determined by GaSe's $\mathrm{D}_{3 h}$ symmetry ${ }^{11}$. This 12 -fold variation is not observed in the cavity-enhanced SHG, where the GaSe crystal only interacts with the evanescent field of the cavity mode, and the angle between the crystal orientation and cavity's electrical field vector is fixed no matter how the laser polarization change. The polarization variation of the pump laser only changes the laser power coupled into the cavity, which therefore induces the four-fold variation of SHG following the function of $\sin ^{6}(2 \theta)$.

The HWP angle-dependences of GaSe's SHGs pumped by the onresonance CW laser and off-resonance pulsed laser indicate axes of the
PC cavity and GaSe's crystal structure, respectively. We could probe the alignment between GaSe's crystal orientation and axes of the PC cavity by comparing them, as shown in Figure $3 \mathrm{c}$. We conclude the Ga-Se bond is aligned to the $y$-axis of the PC cavity with an angle of $\phi=6^{\circ}$, as indicated in Figure $3 \mathrm{~d}$. With this alignment and the nearfields $\left(E_{x}\right.$ and $\left.E_{y}\right)$ of the resonant mode in the GaSe layer, we could calculate the nonlinear polarizations $P_{x}$ and $P_{y}$ generated in GaSe according to its nonlinear susceptibility matrix (Supplementary Information), as shown in Figure 3a. The symmetries of the generated $P_{x}$ and $P_{y}$ indicate the $y$-component of the SHG has a vertical far-field radiation, as well as a high coupling efficiency. Considering the employed cross-polarization setup, the $x$-polarized pump laser and $y$-polarized SHG radiation enable the high efficiency generation and collection of SHG.

The low-power CW pumped SHG in 2D materials is also validated in a mono-layer GaSe. Figure 4a shows the AFM image of an integrated mono-layer GaSe-PC cavity, which has a resonant mode at the wavelength of $1548.8 \mathrm{~nm}$ and a $Q$ factor of $\sim 1800$. By pumping it with a $0.5 \mathrm{~mW}$ on-resonance $\mathrm{CW}$ laser, we measure the vertically scattered upconversion signal, as displayed in Figure 4b. Two clear peaks are observed at wavelengths of 774.4 and $516.3 \mathrm{~nm}$, which are verified as GaSe's SHG and silicon's THG, respectively. Figure 4c and $4 \mathrm{~d}$ plot the pump wavelength and polarization dependences of the SHG signal, yielding similar conclusions as those obtained from the nine-layer GaSe-PC cavity. The results also confirm the cavity enhancement effect via resonant mode's near-field. Because the mono-layer GaSe-PC cavity and the nine-layer GaSe-PC cavity have similar $Q$ factors, the cavity-enhanced THGs of the silicon slab from the two devices should be close. Therefore, we could compare the cavity-enhanced SHG powers from the mono- and nine-layer GaSe by calculating the ratios of GaSe's SHG peak to silicon's THG peak of the

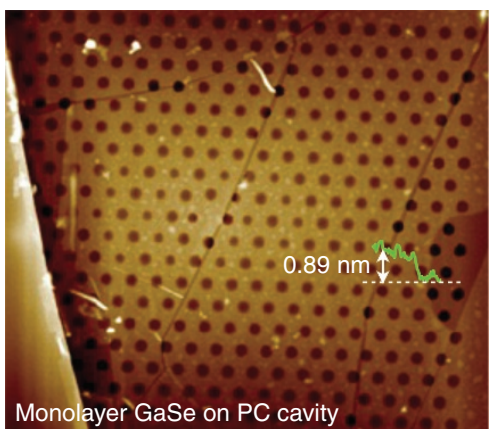

C

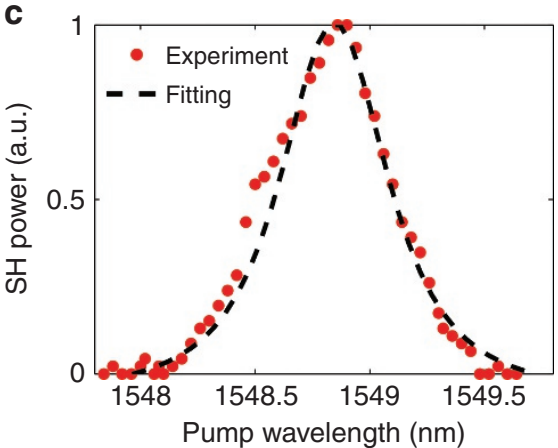

b

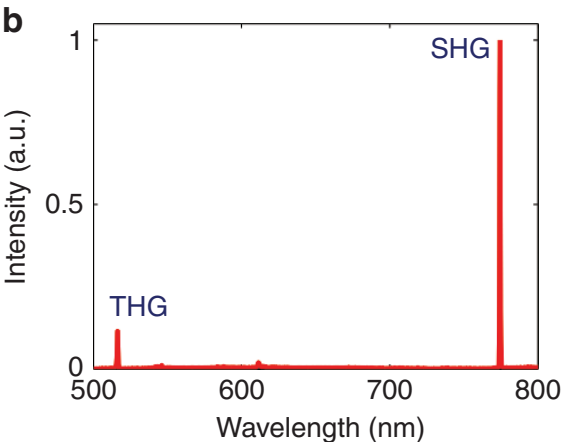

d

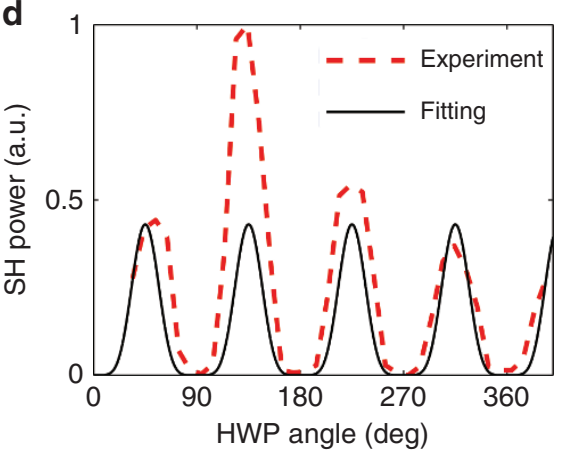

Figure $4 \mathrm{CW}$ pumped SHG from a mono-layer GaSe integrated with a PC cavity. (a) AFM image of the GaSe-PC cavity, indicating GaSe's thickness of $\sim 0.89 \mathrm{~nm}$. (b) Spectrum of the cavity's upconversion signals pumped with a $1548.8 \mathrm{~nm}$ CW laser, showing SHG and THG signals. (c) Cavity-enhanced SHG spectrum when the pump wavelength is tuned cross the resonance, where the dashed line is a squared Lorentzian fitting curve of the cavity resonant mode. (d) Polarization-dependence of the cavity-enhanced SHG. 
two devices separately. From the experimental results, the peak ratios from the mono-layer GaSe-PC cavity and nine-layer GaSe-PC cavity are calculated as 8.7 and 650, that is, SHG from the nine-layer GaSe is about 75 times of that from the mono-layer GaSe. The SHG power variation of the two GaSe flakes is closely consistent with SHG's quadratic dependence on the material thickness for a thin film, as demonstrated in Ref. 28. While the absolute SHG power of the monolayer GaSe is much weaker than that of the nine-layer GaSe, the SHG enhancement factors of the two devices are both around 600, which is determined by the $Q$ factor and mode volume of the cavity mode.

\section{CONCLUSIONS}

In conclusion, we have presented the first demonstration of $\mathrm{CW}$ pumped SHG in 2D materials with the integration of a silicon PC cavity. Assisting by the ultrahigh second order nonlinearity of atomically layered GaSe and extremely confined resonant mode of the cavity, we achieved efficient SHGs in nine- and mono-layer GaSe flakes with an excitation power less than 10 microwatts. Comparing with the SHG pumped by an off-resonance pulsed laser, the onresonance pumped SHG is enhanced by a factor exceeding 600 . The SHG enhancement could be improved further by employing a cavity with even higher $Q$ factor, which is proportional to $Q^{2}$ (Refs 25,29-31). On the other hand, a PC cavity with doubly resonant modes at the fundamental pump and second harmonic signal could achieve a significantly high enhancement as well ${ }^{36}$. The CW pumped SHG could open up opportunities for employing 2D materials' distinct second order nonlinearity to extend their optoelectronic devices in nonlinear regime with low optical power, such as coherent laser frequency converters, upconversion detectors, entangled photon pair generators and so on.

\section{CONFLICT OF INTEREST}

The authors declare no conflict of interest.

\section{ACKNOWLEDGEMENTS}

We thank Kaihui Liu for many fruitful discussions. Financial support was provided by NSFC $(61522507,11404264,61377035,11634010)$, the Natural Science Basic Research Plan in Shaanxi Province of China (2016JQ6004) and the Fundamental Research Funds for the Central Universities (3102017jc01001).

1 Kim K, Choi JY, Kim T, Cho SH, Chung HJ. A role for graphene in silicon-based semiconductor devices. Nature 2011; 479: 338-344.

2 Li LK, Yu YJ, Ye GJ, Ge QQ, Ou XD et al. Black phosphorus field-effect transistors. Nat Nanotechnol 2014; 9: 372-377.

3 Xu MS, Liang T, Shi MM, Chen HZ. Graphene-like two-dimensional materials. Chem Rev 2012; 113: 3766-3798.

4 Shen YR. The Principles of Nonlinear Optics. New York: Wiley-Interscience; 2003.

5 Boyd RW. Nonlinear Optics. 3rd ed. San Diego, CA, USA: Academy Press; 2008.

6 Malard LM, Alencar TV, Barboza APM, Mak KF, de Paula AM. Observation of intense second harmonic generation from $\mathrm{MoS}_{2}$ atomic crystals. Phys Rev B 2013; 87: 201401.

7 Li YL, Rao Y, Mak KF, You YM, Wang SY et al. Probing symmetry properties of few-layer $\mathrm{MoS}_{2}$ and h-BN by optical second-harmonic generation. Nano Lett2013; 13: 3329-3333.

8 Zhao M, Ye ZL, Suzuki R, Ye Y, Zhu HY et al. Atomically phase-matched second-harmonic generation in a 2D crystal. Light Sci Appl 2016; 5: e16131, doi:10.1038/Isa.2016.131.

9 Beams R, Cancado LG, Krylyuk S, Kalish I, Kalanyan B et al. Characterization of fewlayer $1 \mathrm{~T}^{\prime} \mathrm{MoTe}_{2}$ by polarization-resolved second harmonic generation and Raman scattering. ACS Nano 2016; 10: 9626-9636.

10 Janisch C, Wang YX, Ma D, Mehta N, Elías AL et al. Extraordinary second harmonic generation in tungsten disulfide monolayers. Sci Rep 2014; 4: 5530.
11 Zhou X, Cheng JX, Zhou YB, Cao T, Hong $\mathrm{H}$ et al. Strong second-harmonic generation in atomic layered GaSe. J Am Chem Soc 2015; 137: 7994-7997.

12 Yin XB, Ye ZL, Chenet DA, Ye Y, O'Brien K et al. Edge nonlinear optics on a $\mathrm{MoS}_{2}$ atomic monolayer. Science 2014; 344: 488-490.

13 Cheng JX, Jiang T, Ji QQ, Zhang Y, Li ZM et al. Kinetic nature of grain boundary formation in as-grown $\mathrm{MoS}_{2}$ monolayer. Adv Mater 2015; 27: 4069-4074.

14 Seyler KL, Schaibley JR, Gong P, Rivera P, Jones AM et al. Electrical control of secondharmonic generation in a WSe $\mathrm{W}_{2}$ monolayer transistor. Nat Nanotechnol 2015; 10: 407-411.

15 Xie C, Mak C, Tao XM, Yan F. Photodetectors based on two-dimensional layered materials beyond graphene. Adv Funct Mater 2017; 27: 1603886.

16 Ross JS, Klement P, Jones AM, Ghimire NJ, Yan JQ et al. Electrically tunable excitonic light-emitting diodes based on monolayer WSe 2 p-n junctions. Nat Nanotechnol 2014; 9: 268-272.

17 Withers F, Del Pozo-Zamudio 0, Schwarz S, Dufferwiel S, Walker PM et al. WSe 2 lightemitting tunneling transistors with enhanced brightness at room temperature. Nano Lett 2015; 15: 8223-8228.

18 Gan XT, Shiue RJ, Gao YD, Meric I, Heinz TF et al. Chip-integrated ultrafast graphene photodetector with high responsivity. Nat Photonics 2013; 7: 883-887.

19 Pospischil A, Humer M, Furchi MM, Bachmann D, Guider R et al. CMOS-compatible graphene photodetector covering all optical communication bands. Nat Photonics 2013; 7: 892-896.

20 Wang XM, Cheng ZZ, Xu K, Tsang HK, Xu JB. High-responsivity graphene/siliconheterostructure waveguide photodetectors. Nat Photonics 2013; 7: 888-891.

21 Liu M, Yin XB, Ulin-Avila E, Geng BS, Zentgraf T et al. A graphene-based broadband optical modulator. Nature 2011; 474: 64-67.

22 Guo J, Xie JJ, Li DJ, Yang GL, Chen F et al. Doped GaSe crystals for laser frequency conversion. Light Sci Appl 2015; 4: e362, doi:10.1038/lsa.2015.135.

23 Jiang T, Liu HR, Huang D, Zhang S, Li YG et al. Valley and band structure engineering of folded $\mathrm{MoS}_{2}$ bilayers. Nat Nanotechnol 2014; 9: 825-829.

24 Miller S, Luke K, Okawachi Y, Cardenas J, Gaeta AL et al. On-chip frequency comb generation at visible wavelengths via simultaneous second- and third-order optical nonlinearities. Opt Express 2014; 22: 26517-26525.

25 Gan XT, Yao XW, Shiue RJ, Hatami F, Englund D. Photonic crystal cavity-assisted upconversion infrared photodetector. Opt Express 2015; 23: 12998-13004.

26 Guo X, Zou CL, Schuck C, Jung H, Cheng RS et al. A parametric down conversion photon-pair source on a silicon chip platform. Light Sci Appl 2017; 6: e16249, doi:10.1038/lsa.2016.249.

27 Castellanos-Gomez A, Buscema M, Molenaar R, Singh V, Janssen L et al. Deterministic transfer of two-dimensional materials by all-dry viscoelastic stamping. 2D Mater 2014; 1: 011002.

28 Tang YH, Mandal KC, McGuire JA, Lai CW. Layer- and frequency-dependent second harmonic generation in reflection from GaSe atomic crystals. Phys Rev B 2016; 94 : 125302

29 Buckley S, Radulaski M, Petykiewicz J, Lagoudakis KG, Kang JH et al. Secondharmonic generation in GaAs photonic crystal cavities in (111)B and (001) crystal orientations. ACS Photonics 2014; 1: 516-523.

30 Rivoire K, Lin ZL, Hatami F, Masselink WT, Vučković J. Second harmonic generation in gallium phosphide photonic crystal nanocavities with ultralow continuous wave pump power. Opt Express 2009; 17: 22609-22615.

31 Galli M, Gerace D, Welna K, Krauss TF, O'Faolain L et al. Low-power continuous-wave generation of visible harmonics in silicon photonic crystal nanocavities. Opt Express 2010; 18: 26613-26624.

32 Fryett TK, Seyler KL, Zheng JJ, Liu CH, Xu XD et al. Silicon photonic crystal cavity enhanced second-harmonic generation from monolayer WSe 2 . 2D Mater 2016; 4: 015031

33 Watanabe T, Abe H, Nishijima Y, Baba T. Array integration of thousands of photonic crystal nanolasers. App/ Phys Lett 2014; 104: 121108.

34 Gan XT, Pervez N, Kymissis I, Hatami F, Englund D. A high-resolution spectrometer based on a compact planar two dimensional photonic crystal cavity array. Appl Phys Lett 2012; 100: 231104.

35 Gan XT, Mak KF, Gao YD, You YM, Hatami F et al. Strong enhancement of light-matter interaction in graphene coupled to a photonic crystal nanocavity. Nano Lett 2012; 12: 5626-5631.

36 Yi F, Ren ML, Reed JC, Zhu H, Hou JC et al. Optomechanical enhancement of doubly resonant 2D optical nonlinearity. Nano Lett 2016; 16: 1631-1636.

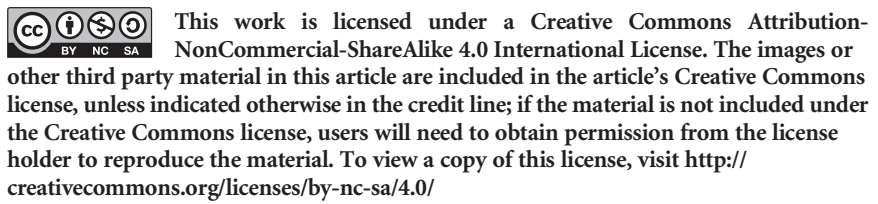

(C) The Author(s) 2018

Supplementary Information for this article can be found on the Light: Science \& Applications' website (http://www.nature.com/lsa). 